\title{
AVANÇOS EM REPRODUÇÃO ASSISTIDA
}

\section{ADVANCES IN ASSISTED REPRODUCTION}

Marcia de Freitas
Arnaldo AF Siqueira
Conceição A M Segre $^{* * *}$

Freitas Mde, Siqueira AAF, Segre CAM. Avanços em Reprodução Assistida. Rev Bras Crescimento Desenvol Hum. 2008; 18(1): 93-97.

Resumo: Buscou-se, por meio de uma revisão bibliográfica de publicações científicas, atualizar conhecimentos produzidos sobre reprodução assistida. Inicialmente, investigou-se o histórico e, em seguida, foram apresentados conceitos relativos à infertilidade e sobre as técnicas de reprodução assistida, especificando-se ainda alguns temas relacionados a aspectos obstétricos, epidemiológicos e perinatais. Vários artigos que abordam o tema foram apresentados. Foi discutida a situação no Brasil bem como os vários aspectos assinalados.

Palavras-chave: Gravidez; gravidez múltipla; infertilidade feminina; infertilidade masculina; fertilização In vitro; transferência embrionária; transferência embrionária/métodos; técnicas reprodutivas.

\section{INTRODUÇÃO}

A Reprodução Humana Assistida (RHA) é, basicamente, a intervenção do homem no processo de procriação natural, com o objetivo de possibilitar que pessoas com problemas de infertilidade e esterilidade satisfaçam o desejo de alcançar a maternidade ou a paternidade.

Ressalta-se que a esterilidade e a infertilidade são doenças devidamente registradas na Classificação Internacional de Doenças - CID $10(\mathrm{OMS})^{(1)} \mathrm{e}$, como tal, podem ser tratadas.

A RHA implica em um conjunto de técnicas a saber: relação programada, inseminação artificial intra-uterina e fertilização extra-corpórea que abrange a fertilização in-vitro clássica e a fertilização in-vitro por meio de injeção intracitoplasmática de espermatozóide. Uma equipe multidisciplinar tem participação estreita no acompanhamento do desenvolvimento folicular, detecção e indução da postura ovular ou até mesmo a realização do encontro dos gametas, assim como na otimização da fase lútea. As indicações dessas técnicas estão diretamente ligadas às causas de infertilidade.

A taxa de sucesso para a avaliação dos resultados das técnicas de RHA implica na ocorrência de gravidez, seja ela bioquímica, gravidez clínica ou o nascimento de uma criança viva.

Assim, o objetivo é verificar os aspectos obstétricos, epidemiológicos e perinatais da Reprodução Humana Assistida (RHA)

\section{MÉTODO}

Pesquisa da literatura médica por meio das bases de dados Medline, EMBASE, SciELo, LILACS e Cochrane Library, usando as palavras-

\footnotetext{
Doutora pela Faculdade de Saúde Pública da Universidade de São Paulo-SP.

Avenida Paulista, 66 apto 52 Bela Vista São Paulo CEP 01310-000 São Paulo Brasil. Email: mardemar@uol.com.br

** Professor Titular do Departamento de Saúde Materno Infantil/ Faculdade de Saúde Pública - USP/SP

*** Livre Docente do Instituto de Ensino e Pesquisa do Hospital Israelita Albert Einstein

Tese apresentada ao Programa de Pós-Graduação em Saúde Pública da Faculdade de Saúde Pública da Universidade de São Paulo para obtenção do título de Doutor em Saúde Pública em 15 de agosto de 2007. Área de Concentração: Saúde Materno-Infantil
} 
chave: gravidez; gravidez, múltipla; infertilidade, feminina; infertilidade, masculina; fertilização In vitro; transferência embrionária; transferência embrionária/métodos; técnicas reprodutivas.

Foram analisados artigos de revisão sistemática, atualização, artigos originais, relatos de caso, capítulos em livros-texto, normas e a legislação vigente no país sobre a temática.

\section{Histórico}

Em 25 de julho de 1978, na Inglaterra, nasceu Louise Toy Brown, o primeiro "bebê de proveta", de fertilização in vitro (FIV) e o evento mais importante até aquele momento em matéria de RHA, que foi realizado por Patrick C. Steptoe e Robert Edwards ${ }^{(2)}$.

Fernandez et al. ${ }^{(3)}$, em 1985, publicaram a ocorrência da primeira gestação obtida por transferência intra tubária de gametas (GIFT), em 1984, no Chile.

Com o auxilio do micromanipulador, surgiu o procedimento de injeção intracitoplasmática de espermatozóide (ICSI), tornando-se até então a mais revolucionaria de todos os tempos. Em 1992 foi descrita na revista Lancet a primeira gestação com o método ${ }^{(4)}$.

Nessa mesma data, um fato revolucionou a comunidade científica e a sociedade: o nascimento da ovelha Dolly, clonada pelo grupo britânico liderado por Ian Wilmut ${ }^{(5)}$.

Em 2007, trabalhos apresentados no $23^{\circ}$. Encontro anual da European Society of Human Reproduction and Embryology (ESHRE), realizado de 1 a 4 de julho de 2007 em Lyon (França) passaram a enfatizar a criopreservação de gônadas, procedimento que representava uma esperança promissora de se obter gestação em mulheres que tivessem, por exemplo, mosaico da síndrome de Turner ${ }^{(6)}$.

\section{RESULTADOS}

\section{Aspectos Obstétricos}

Apesar do reduzido número de estudos que aborda as seqüelas na saúde da mulher pós realização de RHA, há numerosas razões para que sua saúde possa estar comprometida, incluindo os efeitos das drogas utilizadas para a estimulação ovariana, os instrumentos utilizados nos procedimentos de RHA, partos operatórios, entre outros.

A concepção por RHA é associada a um aumento da incidência de uma série de complicações obstétricas e perinatais: perda espontânea de gravidez pré-clinica; aborto espontâneo com uma incidência de 15,5\% nas gestações; gravidez ectópica variável de $0,7 \%$ a 2,2\% dependendo do tipo de procedimento utilizado. Encontrou-se ainda um maior risco de placenta prévia, descolamento prematuro de placenta, além de um maior número de hipertensão arterial especifica da gravidez e diabetes gestacional. Uma outra complicação, embora rara, é a síndrome de superestimulação ovariana que se desenvolve na fase pósovulatória de um ciclo induzido e envolve uma série de complicações potencialmente graves ${ }^{(7)}$.

Relativamente ao tipo de parto, verificouse que o parto cesárea foi muito freqüente entre as gestantes submetidas à RHA, principalmente em função da presença de fetos múltiplos. Há relatos de um possível risco aumentado do câncer ovariano e de mamas em mulheres que receberam a terapia do gonadotrofinas ou com clomifeno ${ }^{(8-9)}$.

\section{Aspectos Perinatais}

Dentre os diferentes aspectos destaca-se o nascimento de mais uma criança, considerado um problema de saúde pública, devido aos riscos causados às mães, às crianças e pelo alto custo gerado ao sistema público de saúde. O maior risco para as crianças é o da prematuridade, em especial a prematuridade extrema. Esses RN apresentam problemas de termorregulação, respiratórios, de alimentação, icterícia, infecção e complicações neurológicas e restrição de crescimento intra-uterino. A longo prazo, verifica-se necessidade de educação especial, dificuldades de comportamento e de socialização ${ }^{(10)}$. A mortalidade perinatal aumenta quatro vezes para a gestação gemelar e seis vezes para a trigemelar.

As taxas mais elevadas de anomalias congênitas são, em sua grande maioria, resultados 
dos efeitos adversos dos nascimentos múltiplos. Nos gêmeos monozigóticos existe uma probabilidade duas a três vezes maior dos defeitos estruturais do que os gêmeos dizigóticos ou os únicos. Acham-se incluídas malformações maiores ou seqüências de malformações, anormalidades causados por alterações vasculares (mais comuns em gêmeos monozigóticos, como a síndrome da transfusão feto-fetal) ${ }^{(11-12)}$.

Katagiri et al ${ }^{(13)}$ em 2007, encontraram taxas elevadas de desordens epigenéticas tais como o síndrome de Angelman e a síndrome de Beckwith-Wiedemann em crianças concebidas por RHA. Os fatores possíveis subjacentes a esses achados incluem características inerentes do gameta, influência da cultura in vitro e peculiaridades dos métodos de RHA. É importante determinar precocemente se tais anormalidades epigenéticas estão presentes nas crianças atualmente concebidas por RHA, para considerar a saúde de gerações seguintes.

\section{Aspectos Epidemiológicos e Demográficos}

Após três décadas de RHA, uma verdadeira "epidemia" de gestações múltiplas vem ocorrendo, em especial nos países desenvolvidos, devido à difusão dessa tecnologia.

Nos Estados Unidos, de 1997 a 2000, a proporção de partos múltiplos atribuíveis à RHA aumentou de $11.2 \%$ para $13.6 \%$, enquanto a proporção atribuível à concepção natural diminuiu de $69.9 \%$ para $64.5 \%{ }^{(14)}$. Considerando-se dados do IBGE ${ }^{(15)}$, pode-se verificar que houve uma alteração na curva demográfica do Brasil quando se compara uma série histórica de nascimentos no período de 1984 a 2003. Enquanto o número de nascimentos, como um todo, elevouse em 9,5\%, o número de triplos ou mais foi cinco vezes maior, no mesmo período. Duas são as explicações possíveis: a interferência dos procedimentos de fertilização assistida e a idade materna mais elevada ${ }^{(16)}$.

Em relação aos gemelares nativivos, há que se assinalar a ocorrência de aumento de $50 \%$ de $\mathrm{RN}$ de baixo peso e de $90 \%$ em gestações triplas, além de um aumento de $\mathrm{RN}$ com restri- ção de crescimento intra-uterino e suas complicações, como a ocorrência de doenças crônicas da criança e do adulto ${ }^{(7,17-18)}$

\section{Situação da RHA no Brasil}

Dos 117 centros de RHA brasileiros, apenas seis Instituições oferecem o tratamento totalmente gratuito e três oferecem o serviço gratuitamente, mas com a medicação paga pelo casal. Há ainda um serviço público de infertilidade no Rio de Janeiro, que disponibiliza apenas os exames para diagnosticar a causa da infertilidade ${ }^{55}$. Essa situação ainda se agrava quando se observa que além do insuficiente número de centros de RHA públicos para a demanda, $80 \%$ desses estão concentrados no Estado de São Paulo e distribuídos de maneira não equânime no próprio Estado e no País.

Um fator preocupante no Brasil é o fato de que as técnicas de reprodução assistida são regulamentadas apenas pelas normas éticas definidas pela resolução do Conselho Federal de Medicina ${ }^{(19)}$. Essa falta de legislação no País, em especial relacionada ao número de pré embriões transferidos, fez com que as gestações múltiplas, aumentassem exponencialmente no Brasil, gestações essas consideradas um problema de saúde pública, devido aos riscos causados às mães, às crianças e pelo alto custo imposto ao sistema público de saúde ${ }^{(20)}$.

\section{CONSIDERAÇÕES FINAIS}

Os aspectos epidemiológicos, obstétricos e perinatais da RHA devem ser discutidos em profundidade não somente pelas Sociedades Médicas das especialidades envolvidas, como também pela sociedade em geral em seus diversos segmentos.

Apesar de todos os benefícios que a RHA tem trazido para casais inférteis, ainda são necessários muitos estudos para que se possa estabelecer um consenso a respeito. Pode se dizer que "o admirável mundo novo da RHA" trouxe repercussões que configuraram uma nova realidade a ser avaliada em toda a sua magnitude. 


\begin{abstract}
This study aimed to survey the knowledge produced on human assisted reproduction, by means of a bibliographic review of scientific publications. First, we investigated the history; then, we presented concepts related to infertility and to the techniques used in human assisted reproduction. Some other themes were detailed, such as those involving obstetric, epidemiological, and perinatal aspects. Several studies that deal with the theme were presented. The situation of human assisted reproduction in Brazil was discussed, as well as the various aspects mentioned above.
\end{abstract}

Keywords: Pregnancy; multiple pregnancy; female infertility; male infertility; in vitro fertilization; embryo transfer; embryo transfer/methods; reproductive techniques.

\section{REFERÊNCIAS}

1. CDC National Center for Health Statistics. Infertility. Disponível em: http://www.cdc.gov/ nchs/datawh/nchsdefs/infertility.htm Acessado 2008 março 15.

2. Steptoe PC, Edwards RG. Birth after reimplantation of human embryo. Lancet. 1978; 2(8085):366.

3. Fernandez E, Zegers F, Hess R, Roblero L, Ortiz ME, Guadarrama A, et al. Transferencia intratubaria de gametos (TITG, GIFT): comunicación del primer embarazo. Rev Chil Obstet Ginecol. 1985; 50(5):35662.

4. Palermo G, Joris H, Devroey P. Van Steirtegehm AC. Pregnancies after intracitoplasmatic injection of single spermatozoon into oocyte. Lancet. 1992; 340(8810):17-9.

5. Wilmut I, Schnieke AE, McWhir J, Kind AJ, Campbell KHS. Viable offspring derived from fetal and adult mammalian cells. Nature. 1997; 385: 810 -3 .

6. ESHRE. 23 rd Annual Meeting of the European Society of Human Reproduction and Embriology.[texto na Internet] 2007. Disponível em: http://humrep.oxfordjournals.org/content/vol22/ suppl_1/index.dtl Acessado em 2007 junho 10.

7. CDC-Centers for Disease Control. Assisted Reproductive Technology. 2007. [texto na Internet] 2006. Disponível em: http://www.cdc.gov/art/ Acessado em 2008 janeiro 10

8. Brinton LA, Lamb EJ, Moghissi KS, Scoccia B, Althuis M, Mabie J, Westhoff C. Ovarian cancer risk associated with varying causes of infertility. Fertil Steril. 2004; 82:405-14.

9. Burkman RT, Tang MC, Malone KE, Marchbanks PA, McDonald JA, Folger SG, et al. Infertility drugs and the risk of breast cancer: findings from the National Institute of Child Health and Human Development Women's Contraceptive and Reproductive Experiences Study. Fertil Steril. 2003; 79(4):844-51.
10. Reddy UM, Wapner RJ, Rebar RW, Tasca RJ. Infertility, assisted reproductive technology, and adverse pregnancy outcomes: executive summary of a National Institute of Child Health an Human Development workshop. Obstet Gynecol. 2007; 109(4): 967-77.

11. Jones KL. Smith's recognizable patterns of human malformation. 6th ed. Philadelphia: Elsevier Saunders; 2006.

12. Schinzel AA, Smith DW, Miller JR. Monozygotic twinning and structural defects. J Pediatr. 1979; 95(6):921-30.

13. Katagiri Y, Shibui Y, Nagao K, Miura K, Morita M. Epigenetics in assisted reproductive technology. Reprod Med Biol. 2007; 6(2): 69-75.

14. Reynolds MA, Schieve LA, Martin JA, Jeng G, Macaluso M.Trends in multiple births conceived using assisted reproductive technology, United States, 1997-2000. Pediatrics. 2003;111(5 Part 2):1159-62.

15. IBGE Instituto Brasileiro de Geografia e Estatística. Sidra - Tabela 2466 - pessoas de 10 anos ou mais por estado civil, situação de domicilio, condição de convivência e grupos. [texto na Internet] 2006. Disponível em http://www.sidra.ibge.gov.br/bda/ tabela/listabl.asp?z=t\&c=2466 Acessado em 2008 janeiro10.

16. Colletto GMDD, Segre CAM, Rielli STRC Horácio MR. Multiple birth rates according to different socioeconomic levels: an analysis of four hospitals from the city of Sao Paulo, Brazil. Twin Res.2003; 6(3): 177-82.

17. Wright VC, Shieve LA, Reynolds MA, Jeng G. Assisted reproductive technology surveillance. MMWR 2005/ 54 (SS02); 1-24. [texto na Internet] 2005. Disponível em: http://www.cdc.gov/mmwr/ preview/mmwrhtml/ss5402a1.htm Acessado em 2008 março 10.

18. Kansal-Kalra S, Milad MP, Grobman WA. In vitro fertilization (IVF) versus gonadotropins followed by IVF as treatment for primary infertility: a cost- 
based decision analysis. Fertil Steril. 2005; 84(3):600-4.

19. Brasil. Ministério da Saúde. Direitos Sexuais e Direitos Reprodutivos: uma prioridade do governo. Série A. Normas e Manuais Técnicos. Brasília, DF, $2005.24 \mathrm{p}$.
20. Diniz D. Tecnologias reprodutoras, ética e gênero: o debate legislativo brasileiro. In: Barchifontaine CP, Pessini L.(editores). Bioética: alguns desafios. São Paulo: Loyola;2001.p.203-24.

Recebido em: 06/10/2007

Modificado em: 10/02/2008

Aprovado em: 11/03/2008 